\title{
Automatic segmentation of juxta-pleural tumors from CT images based on morphological feature analysis
}

\author{
Jin Rim Yong ${ }^{\text {a,b,c }}$, Shouliang Qi ${ }^{\text {b,c, }}$, Han J.W. van Triest ${ }^{\text {b,c }}$, Yan Kang ${ }^{\text {b,c }}$ and Wei Qian ${ }^{\text {b,d }}$ \\ ${ }^{a}$ Computer Science Department, Kim Il Sung University, Pyongyang, DPR Korea \\ ${ }^{b}$ Sino-Dutch Biomedical and Information Engineering School, Northeastern University, Shenyang \\ 110000, China \\ ${ }^{c}$ Key Laboratory of Medical Imaging Computing (Ministry of Education), Northeastern University, \\ Shenyang 110000, China \\ ${ }^{d}$ College of Engineering, University of Texas, El Paso, TX, USA
}

\begin{abstract}
Extraction of lung tumors is a fundamental step for further quantitative analysis of the tumor, but is challenging for juxta-pleural tumors due to the adhesion to the pleurae. An automatic algorithm for segmentation of juxta-pleural tumors based on the analysis of the geometric and morphological features was proposed. Initially, the lung is extracted by means of thresholding using 2D Otsu's method. Next a center point is suggested to find a starting point and endpoint of outward facing pleura. A model based on the variation of incline angle was adopted to identify potentially affected regions, and to full segment juxta-pleural tumors. The results were compared with the manual segmentation by two radiologists. Averaged for ten experimental datasets, the accuracy calculated by Dice index between the results of the algorithm and by the two radiologists is $91.2 \%$. It indicates the proposed method has comparable accuracy with the experts (the inter-observer variability is $92.4 \%$ ), but requests much less manual interactions. The proposed algorithm can be used for segmenting juxta-pleural tumors from CT images, and help improve the diagnosis, pre-operative planning and therapy response evaluation.
\end{abstract}

Keywords: Juxta-pleural tumor segmentation, morphological analysis, incline angle

\section{Introduction}

Segmentation of lung cancer from CT images is a crucial and challenging task, and is an important pre-condition for computer-aided diagnosis systems. In recent years, various methods have been proposed for lung and lung tumor segmentation and recognition including thresholding methods, deformable boundary-based methods, shape-based methods and edge-based methods [1].

Among the segmentation techniques, automatic thresholding methods are most widely used, owing to their simple implementation and low time complexity [2]. In [3], an iterative thresholding-based technique is introduced to acquire an initial lung area. The morphological opening and closing opera-

${ }^{*}$ Corresponding author: Shouliang Qi, Sino-Dutch Biomedical and Information Engineering School, Northeastern University, Shenyang 110000, China. Tel.: (86)-24-83680299; Fax: (86)-24-83681955; E-mail: qisl@bmie.neu.edu.cn.

0959-2989/14/\$27.50 @ 2014 - IOS Press and the authors. 
tions were used to refine this initial segmentation. In [4], an adaptive fuzzy threshold is used to segment the lungs from CT data followed by smoothing of the segmented lung contour through Gaussian smoothing.

Besides thresholding, active contour models have been applied extensively to image segmentation. There are several desirable advantages of active contour models over classical image segmentation methods, such as edge detection and region growing methods [5]. In [6], a 2D parametric deformable model approach is employed to extract the lung fields. In their framework, the deformable boundary is initialized based on a threshold estimated from CT data, and the lung borders are used as an external force to constrain the deformable model evolution. In [7], the lungs were segmented using a 3D Active Shape Model (ASM) matching process and a globally optimal surface finding method to obtain a refined and smoothed segmentation.

Lung tumors often distort the surrounding anatomical structures such as the pleural surface and vasculature. It is especially true for juxta-pleural tumors where a significant portion of the neoplasm is connected to the pleural surface [8]. Several methods have been suggested to detect the juxta-pleural pathological areas in chest CT images. In $[9,10]$, the methods are proposed that used a boundary correction algorithm based on the convex hull operation and ray casting. Its novelty lies in the fact that it corrects the lung border in a geometric way and can reliably include juxta-pleural nodules and pulmonary vessels. Kim et al. [11] used a contour-following method that tracked the segmented lung boundaries to detect a seed area with texture features of an actual juxta-pleural nodule. However, texture features alone do not sufficiently define juxta-pleural nodules. Tumors can be segmented more accurately by their position and geometric features. Gurcan et al. [12] designed a method for the indentation detection that computes the ratio between the geodesic distances of two points on the border and the Euclidean distance of the line segment formed by the same two points. Korfiatis et al. [13] combined the wavelet edge highlighting and a 3D grey level thresholding using minimum error technique [14]. Moreover, level set model had been considered an important kinds of methods to lung tumor segmentation $[15,16]$.

In this paper, an automated lung and juxta-pleural tumor segmentation algorithm is proposed, using morphological feature analysis of lung wall and shape analysis-based methods. A point of reference is introduced that is used in the morphological features analysis of the lung wall, named "center point" in this work.

\section{Materials and methods}

\subsection{Initial segmentation of lung boundary and selection of a center point}

At first, two potential thresholds $\left(\mathrm{T}_{1}\right.$ and $\left.\mathrm{T}_{2}\right)$ are automatically determined from histogram analysis of CT images. As shown in Figure 1, $\mathrm{T}_{1}$ is the minimum value in concave section between of two high convex, and $\mathrm{T}_{2}$ is the point owing the minimum gradient on the right half boundary of the second convex. Based on $\mathrm{T}_{1}$ and $\mathrm{T}_{2}$, two optimal thresholds $\left(\mathrm{T}_{1 \mathrm{~m}}\right.$ and $\left.\mathrm{T}_{2 \mathrm{~m}}\right)$ can be obtained according to $2 \mathrm{D}$ Otsu's method. The 2D-Otsu method has a better performance, especially for images with low SNR and low contrast, by utilizing the gray level and spatial information of the pixels [17].

$\mathrm{T}_{1 \mathrm{~m}}$ is used to segment the lung field and get the lung boundary. $\mathrm{T}_{2 \mathrm{~m}}$ is aimed to get the point in vertebrae region with the highest vertical coordinate, named "center point". Due to the anatomical nature, this vertebrae area usually appears near the center in the lung CT image. From the binary image 
obtained by thresholding of $\mathrm{T}_{2 \mathrm{~m}}$, the center point is used to determine the endpoint of the lung boundary in the resampling of lung wall. The point is obtained through the following process:

1. Obtain coordinates of all objects (i.e. all white connected sets) in the binary image.

2. Obtain objects in the center band of the binary image.

3. Examine the size (i.e., number of coordinates) in these objects, and select the largest object.

4. Obtain the top point coordinate of the selected object; this point will be referred to as the center point (Figure 2).

\subsection{Resampling of the lung boundary}

In this section, the lung boundaries of pleura are divided evenly into smaller parts (nodes) with equal length for incline angle analysis of each node. The lung boundary resampling is performed from the point with the highest $y$-coordinate of each pulmonary boundary to the nearest point of the outside boundary from the center point. In this paper, the resampling interval was set to 20 pixels based on experimental results. Several lung boundaries that were broken by this method are shown in Figure 3 .

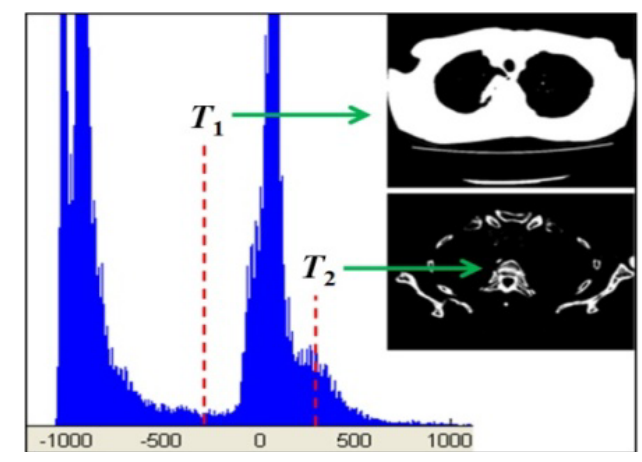

Fig. 1. Selection of thresholds in histogram of CT image and binary images by thresholding, $\mathrm{T}_{1}$ is used for initial pulmonary area segmentation, and $\mathrm{T}_{2}$ is used for selection of a center point.

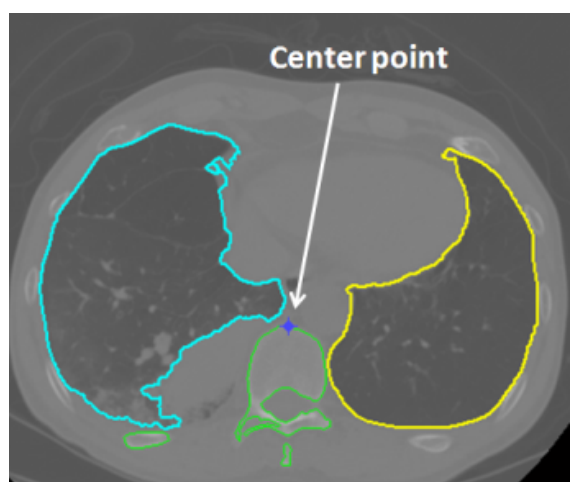

Fig. 2. Initial pulmonary boundary and center point. 

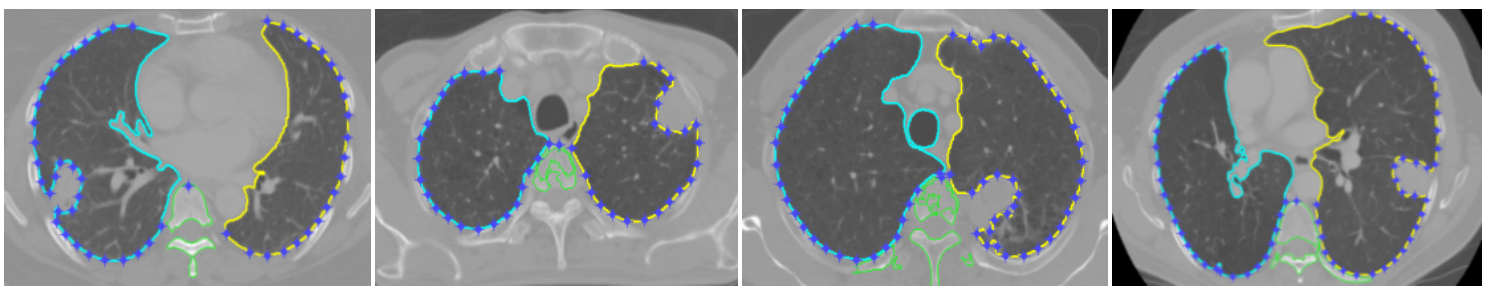

Fig. 3. Resampling of the lung boundary in several CT images.

(a)
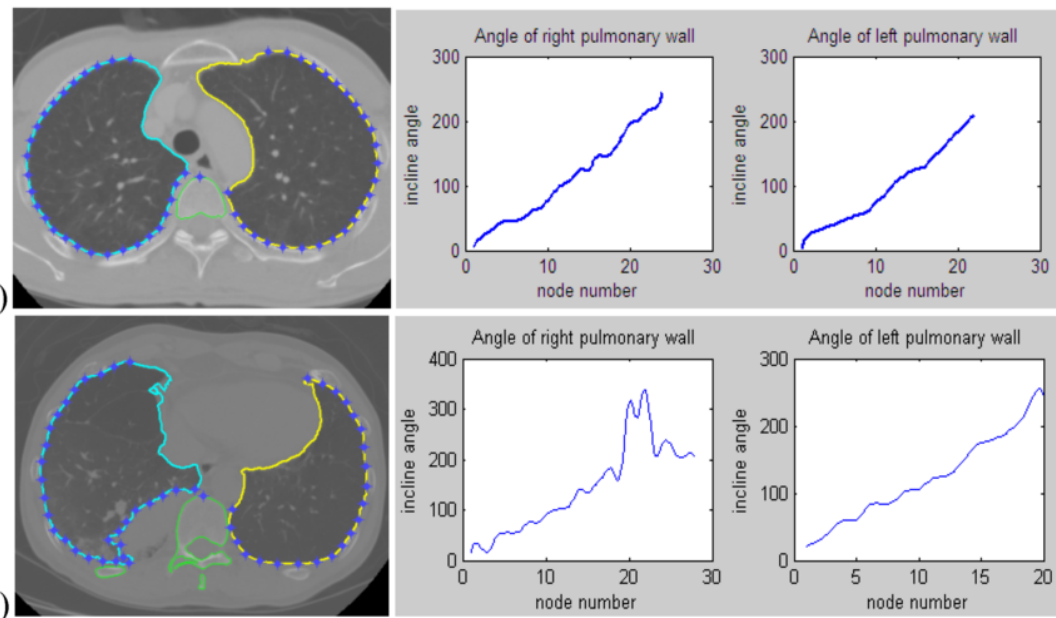

Fig. 4. Analysis of incline angle on chest CT images. (a) Incline angle for regular CT lung boundary, (b) Incline angle in a pathological lung.

\subsection{Morphological feature analysis of lung wall}

In the resampled lung boundary, the incline angle in each node is studied. In here, the incline angle $(\alpha)$ of a node is defined as the value of the angle between the line segment of two consecutive points on the edge and a reference direction. The incline angle of each node is calculated using the following expression.

$$
\alpha_{i}=\arctan \left(\left(\mathrm{x}_{i+1}-\mathrm{x}_{i}\right) /\left(\mathrm{y}_{i+1}-\mathrm{y}_{i}\right)\right)
$$

where $\left(\mathrm{x}_{i}, \mathrm{y}_{i}\right)$ is the coordinate of resampling points in the each lung wall.

In the investigation on typical CT images, the incline angle is relatively even and constant (Figure 4.a). Analysis of pathological CT images shows a more complicated pattern due to the variation in the shape, as shown in Figure 4(b).

\subsection{Automatic localization and segmentation of juxta-pleural tumor areas}

\subsubsection{Finding of juxta-pleural tumor area}

An important portion of the lung segmentation process is to identify the regions with increased changes in their incline angles. In this section, the areas where the changes occur are identified by means of a simple threshold $\mathrm{T}_{\text {angle }}$ : 


$$
\mathrm{T}_{\text {angle }}=\left(\sum_{i=1}^{n-1}\left|\alpha_{i+1}-\alpha_{i}\right|\right) / 2
$$

where $n$ is number of nodes, $\alpha_{i}$ is the incline angle in each node, $\mathrm{T}_{\text {angle }}$ is used to acquire start (or begin) and end points of sections with excessive change.

$$
\mathrm{P}_{\text {begin }}=\left\{i \mid \alpha_{i}-\alpha_{i-1}>\mathrm{T}_{\text {angle }}(i=1 \sim \mathrm{n})\right\}
$$

The end point of a section with excessive change is identified using the following conditions:

$$
\mathrm{P}_{\text {end }}=\left\{i-1 \mid \alpha_{j}-\alpha_{j-1}(j=i \sim i+3)<\mathrm{T}_{\text {angle }}\right\}
$$

While selecting the end point of change section, the node interval between of $\mathrm{P}_{\text {begin }}$ and $\mathrm{P}_{\text {end }}$ has to be more than two nodes.

\subsubsection{Segmentation of juxta-pleural tumor area}

The juxta-pleural tumor sections are identified on the initial pulmonary boundary. The tumor is separated from the wall by forecasting the lung shape. In this paper, the following method for the prediction of the actual lung shape is used. First, the prediction angle in the starting location of the section with change is computed, and the further angles are extrapolated from that to arrive at the end point of the suspected section, see Figure 5.

\section{Experimental result and analysis}

In this section, the proposed algorithm is evaluated by means of experiments. For the evaluation, 50 abnormal cases containing at least one juxta-pleural nodule per case were selected. Figure 6 shows several examples of the separated nodes and their inclination angles.

The proposed method is analyzed compared to the manual segmentation by two experienced radiologists. The analysis results are shown in Table 1.

(a)

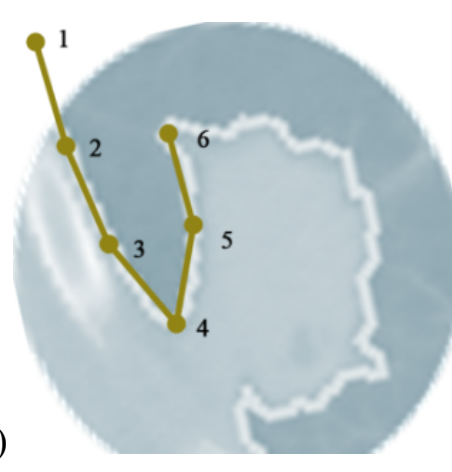

(b)

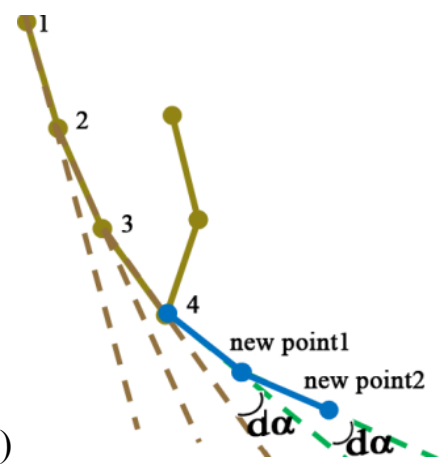

Fig. 5. Prediction of the next node of the pulmonary boundary in the juxta-pleural tumor area, (a) Initial resampling nodes, (b) Prediction process. 

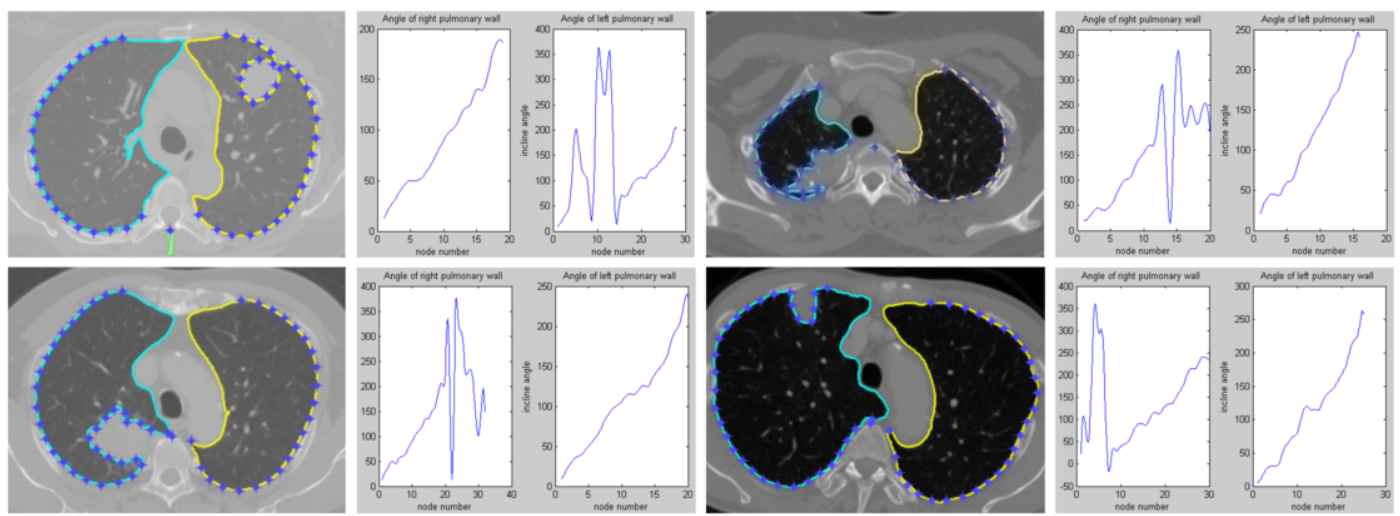

Fig. 6. Resampling nodes and their incline angles.

Table1

Dice index of the results between the algorithm and radiologists

\begin{tabular}{|l|l|l|l|l|l|l|l|l|l|}
\hline No & $\begin{array}{l}\text { Algorithm } \\
\text { (pixel num- } \\
\text { ber) }\end{array}$ & $\begin{array}{l}\text { Radiologist } \\
\text { (pixel } \\
\text { number) }\end{array}$ & $\begin{array}{l}\text { Overlap1 } \\
\text { (pixel num- } \\
\text { ber) }\end{array}$ & $\begin{array}{l}\text { Dice index1 } \\
(\%)\end{array}$ & $\begin{array}{l}\text { Radiologist } \\
\text { (pixel } \\
\text { number) }\end{array}$ & $\begin{array}{l}\text { Overlap2 } \\
\text { (pixel num- } \\
\text { ber) }\end{array}$ & $\begin{array}{l}\text { Dice index2 } \\
(\%)\end{array}$ & $\begin{array}{l}\text { Evaluate } \\
\text { accuracy } \\
(\%)\end{array}$ & $\begin{array}{l}\text { Radiologist } \\
1 / \text { Radiolo- } \\
\text { gist 2 }(\%)\end{array}$ \\
\hline 1 & 3134 & 3133 & 2960 & 94.46 & 3107 & 2741 & 87.84 & 91.15 & 90.87 \\
\hline 2 & 1462 & 1591 & 1448 & 94.86 & 1483 & 1357 & 93.65 & 94.26 & 96.29 \\
\hline 3 & 1674 & 1497 & 1462 & 92.21 & 1551 & 1403 & 87.01 & 89.61 & 92.98 \\
\hline 5 & 1686 & 1594 & 1603 & 97.74 & 1752 & 1572 & 91.45 & 94.60 & 96.41 \\
\hline 6 & 1543 & 1634 & 1458 & 91.78 & 1757 & 1475 & 89.39 & 90.59 & 93.31 \\
\hline 7 & 2612 & 2638 & 2612 & 99.5 & 2842 & 2609 & 95.67 & 97.59 & 94.49 \\
\hline 8 & 2662 & 745 & 688 & 88.15 & 785 & 683 & 85.32 & 86.74 & 95.61 \\
\hline 9 & 805 & 659 & 2617 & 98.75 & 2848 & 2609 & 94.7 & 96.73 & 94.97 \\
\hline 10 & 2946 & 2691 & 2349 & 83.34 & 2896 & 2631 & 90.07 & 86.71 & 87.81 \\
\hline Ave & - & & -641 & 87.57 & 610 & 570 & 80.57 & 84.07 & 82.58 \\
\hline
\end{tabular}

Table1 shows experimental results of 10 cases of juxta-pleural lung tumors. Segmented tumor area is described by the number of pixels. The results are evaluated using the Dice index defined below.

$$
\text { Dice index }=\left(\frac{2 *(A \cap B)}{A+B}\right) * 100 \%
$$

It is found that, averaged for ten experimental datasets, the accuracy calculated by Dice index between the results of the algorithm and by the radiologists is $91.2 \%$. It indicates the proposed method has comparable accuracy with the experts (the inter-observer variability is $92.4 \%$ ), but requests much less manual interactions.

\section{Discussion}

In this paper, a new segmentation method for juxta-pleural tumors based on analysis of the incline angle of pulmonary wall is presented and a prediction model for the lung form is proposed. The seg- 
mentation algorithm of the juxta-pleural tumor area based in the incline angle analysis of the lung boundary suggested in this paper is widely applicable to CT images. In this present study, only images of an axial plane in the lung CT volume have been investigated and evaluated. Future work will include improvements to this process and expansion to 3D volumes, and build an efficient CAD system for medical diagnosis and treatment.

\section{Conclusion}

In this paper, the following problems were addressed:

a. In the initial segmentation of chest CT image, the method of generating the candidate threshold is proposed to reduce the computational complexity.

b. A center point is proposed to determine the endpoint of lung boundary.

c. According to the incline angle change of a lung wall, an automatic localization and segmentation method for juxta-pleural tumor areas is proposed.

$\mathrm{d}$. The tumor area is segmented using a prediction model on based shape analysis of the lung wall.

This automated juxta-pleural tumor localization and segmentation method utilizes CT intensity distribution and morphological features. Comparisons between the results from the algorithm and the radiologists have shown a Dice coefficient of $91.2 \%$ in average.

\section{Acknowledgment}

This work was financially supported by the Natural Science Foundation of Liaoning Province (No. 2013020219), the Scientific Research Fund of Liaoning Provincial Education Department (L2012080), and the Fundamental Research Funds for the Central Universities (N130219001).

\section{References}

[1] A. Soliman, F. Khalifa, A. Alansary, G. Gimel'farb and A. El-Baz, Segmentation of lung region based on using parallel implementation of joint MGRF: Validation on 3D realistic lung phantoms, 2013 IEEE 10th International Symposium on Biomedical Imaging (ISBI), 2013, 864-867.

[2] Jun Zhang and Jinglu Hu, Image segmentation based on 2D Otsu method with histogram analysis, 2008 International Conference on Computer Science and Software Engineering, 2008, 105-108.

[3] S. Hu, E. Hoffman and J. Reinhardt, Automatic lung segmentation for accurate quantitation of volumetric X-ray CT images, IEEE Trans. Med. Imag. 20 (2001), 490-498.

[4] X. Ye, X. Lin, J. Dehmeshki, G. Slabaugh and G. Beddoe, Shape-based computer-aided detection of lung nodules in thoracic CT images, IEEE Trans. Biomed. Eng. 56 (2009), 1810-1820.

[5] C.M. Li, C.Y. Kao, J.C. Gore and Z.H. Ding, Minimization of region-scalable fitting energy for image segmentation, IEEE Transactions on Image Processing 17 (2008), 1940-1949.

[6] Y. Itai, K. Hyoungseop, S. Ishikawa, S. Katsuragawa, T. Ishida, K. Nakamura and A. Yamamoto, Automatic segmentation of lung areas based on SNAKES and extraction of abnormal areas, IEEE Int. Conf. Tools with Artifitial Intell. ICTAI'05, 2005, 377-381.

[7] S. Sun, C. Bauer and R. Beichel, Automated 3-D segmentation of lungs with lung cancer in CT data using a novel robust active shape model approach, IEEE Trans. Med. Imag. 31 (2012), 449-460.

[8] W.J. Kostis, A.P. Reeves, D.F. Yankelevitz and C.I. Henschke, Three dimensional segmentation and growth-rate estimation of small pulmonary nodules in helical CT images, IEEE Transactions on Medical Imaging, 2003, 1259-1274.

[9] Guanglei Si, Jinfeng Cai and Yan Kang, A three dimensional ray casting method for juxta-pleural nodule segmentation in thoracic CT images, 2012 International Conference on Information and Automation (ICIA), 2012, 230-235. 
[10] A.A. Farag, H. Abdelmunim, J. Graham, S. Elshazly, S. El-Mogy, R. Falk, S. Al-Jafary, H. Mahdi and R. Milam, Variational approach for segmentation of lung nodules, Image Processing (ICIP), 2011, 2157-2160.

[11] D.Y. Kim, J.H. Kim, S.M. Noh and J.W. Park, Pulmonary nodule detection using chest CT images, Act. a Radiol. 44 (2003), 252-257.

[12] M.N. Gurcan, B. Sahiner, N. Pertrick, H.P. Chan, E.A. Kazerooni, P.N. Cascade et al., Lung nodule detection on thoracic computer tomography images: preliminary evaluation of a computer-aided diagnosis system, Medical Physics 29 (2002), 2552-2558.

[13] P. Korfiatis, S. Skiadopoulos, P. Sakellaropoulos, C. Kalogeropoulou and L. Costaridou, Combining 2D wavelet edge highlighting and 3D thresholding for lung segmentation in thin-slice CT, Br. J. Radiol. 80 (2007), 996-1004.

[14] J. Kittler and J. Illingworth, Minimum error thresholding, Pattern Recognition 19 (1986), 41-47.

[15] A. Amutha and R.S.D. Wahidabanu, Lung tumor detection and diagnosis in CT scan images, 2013 International Conference on Communications and Signal Processing (ICCSP), 2013, 1108-1112.

[16] Joseph Awad et al., Three-dimensional lung tumor segmentation from x-ray computed tomography using sparse field active models, Medical Physics 39 (2012), 851-865.

[17] R. Helen, N. Kamaraj, K. Selvi and V. Raja Raman, Segmentation of pulmonary parenchyma in CT lung images based on 2D Otsu optimized by PSO, Proc. Emerging Trends in Electrical and Computer Technology (ICETECT), 2012, 536541 